\title{
Current state of communication systems based on electrical power transmission lines
}

Antony Ndolo ${ }^{1,2^{*}}$ (] and İsmail Hakkı Çavdar ${ }^{1}$

\section{*Correspondence:}

394184@ogr.ktu.edu.tr

${ }^{1}$ Department of Electrical

and Electronics Engineering,

Karadeniz Technical

University, Trabzon, Turkey

Full list of author information

is available at the end of the

article

\begin{abstract}
Power line communication technology is a retrofit alternative technology for last mile information technology. Despite several challenges, such as inadequate standards and electromagnetic compatibility, it is maturing. In this review, we have analysed these obstacles and its current application status.
\end{abstract}

Keywords: Electrical power lines, Power line communication (PLC), Power line networks, Noise, Channels, Modulation

\section{Introduction}

Indeed, advancements in communication engineering and technology have brought in revolution in the telecommunication industry. One great impact has been in information and service delivery during the last decades of the twentieth century to date. This is due to the high demand for information created by the huge human population. Better methods and channel models for signal transmission have been researched and developed. For instance, fibre optics has provided waveguide for numerous services at higher speed while inheriting other advantages such immunity to electromagnetic interferences amongst others $[1,2]$. Despite all the positive attractions towards fibre communication, it is expensive to install and it is limited to certain areas. That is, remote, rural and mountainous areas. This has necessitated the search for alternative information transmission methods. Power line communication (PLC) is one such alternative.

Power line communication technology is the basically a technology that uses preexisting and installed electrical power cables for transmission of information [3-6]. Traditionally, such electrical lines were designed exclusively for distribution and transmission of electricity at lower frequency. This frequency varies from country to country, mainly, $50 \mathrm{~Hz}$ or $60 \mathrm{~Hz}$. Upon generation of electricity, it is distributed and transmitted through different voltage network. Firstly, electricity is transmitted over high voltage lines, then distribution is done over medium voltage lines, and lastly, it is converted/ scaled down using transformers for the end-user consumption in the low-voltage lines. Figure 1 gives summary of PLC structure. This technology is therefore retrofit and economically cheaper compared to other methods. There is no need for new cable installations. Secondly, electrical power network is the most developed, covers large areas and 


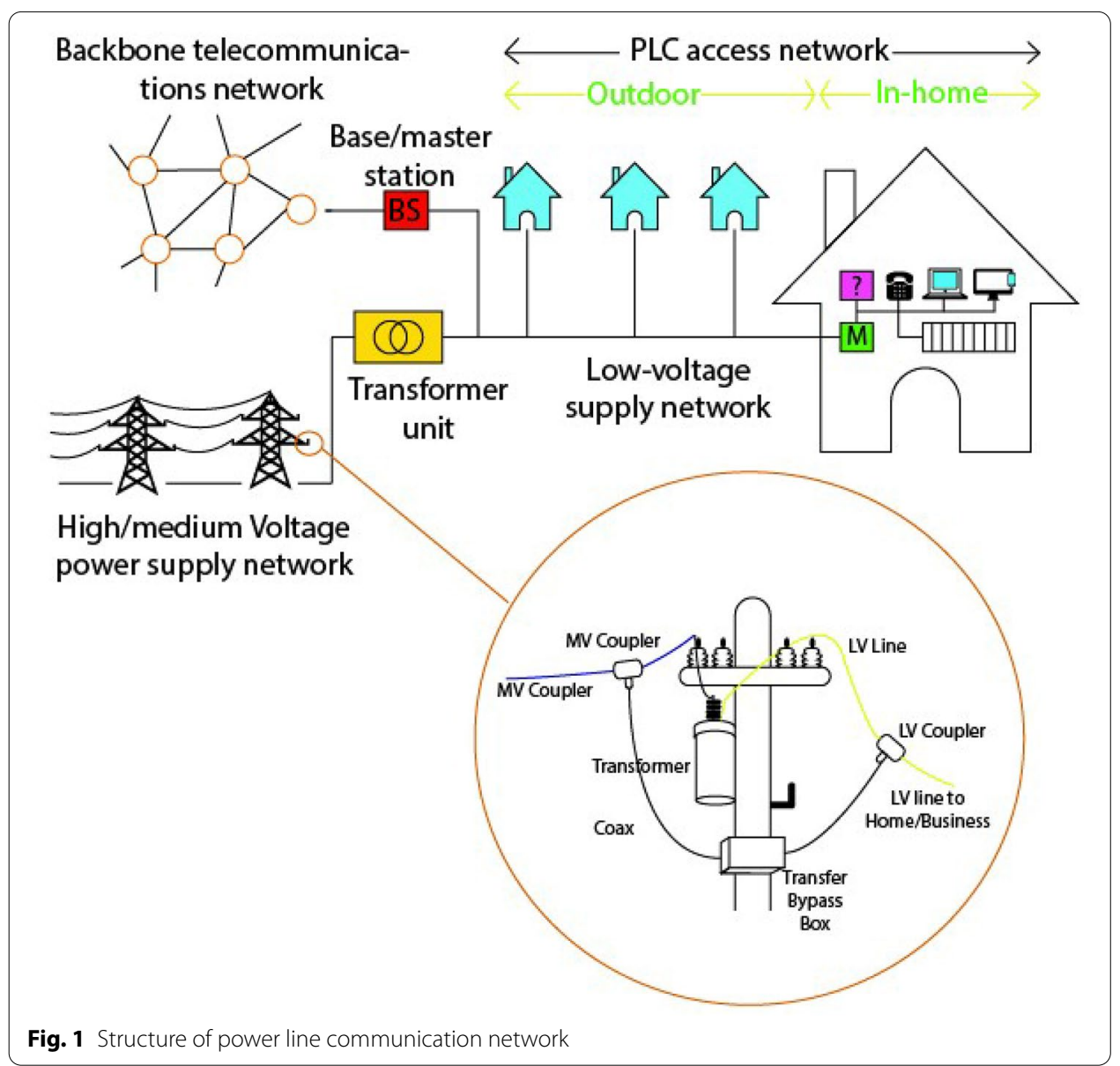

reaches many homesteads. At distribution lines, they are majorly used for the control signals, remote data acquisition and IP telephony services [7].

Power line communication is divided into three categories, namely ultra-narrowband, narrowband and broadband as summarised in Table 1. The first two are commonly grouped together and termed as narrowband PLC. We will characterise these categories in the next section in details.

\section{Classification of power line communication}

The initial idea to use electrical power line for communication purposes was first put foward in the early 1900s. Schwartz in [8] gives comprehensive history regarding the technology. In the paper, PLC evolution in upto the early 2000s is also reviewed. According to [9], we have three categories of PLC as mentioned in the previous section. They are classified based on frequency band of operation and application areas. In Table 1, we give a brief summary of each class. Each category varies in application areas. For instance, ultra-narrowband PLC has been applied in automatic metre reading technology, while the second type has found its application in advance metre reading [10], electric vehicles [11], smart grid [9, 12] and street lighting [13, 14].

Broadband PLC has penetrated to high-speed internet access and home area network applications including audio, HDTV, online gaming and others in $[17,18]$. 


\section{PLC regulation standards}

For universal functionality, there is need for proper standards to regulate communication quality and as well as to cater for minimal, if not zero, interference to human health. In telecommunication, such standards aid in designing an optimal communication system. The parameters that require governing and continuous check include signal-tonoise ratio (SNR) and bit-error-rate (BER). There has been an ever-rising interest in PLC technology in the last two decades. Therefore, researchers and non-governmental bodies have come up with set of rules and requirements to improve optimality and interoperability. This is of course to make electrical power lines more compatible and secure for transmission of different varieties of signals.

Internationally, the following organisations are responsible for setting and governing standards in the telecommunication industry; International Telecommunication Union (ITU), European Committee for Electro-technical Standardisation (CENELEC), International Electro-technical Commission (IEC), Institute of Electrical and Electronics Engineers (IEEE), International Organisation for Standardisation (ISO) and lastly, Federal Communication Commission's (FCC). All these organisations have set standards for both broadband and narrowband PLC. Nonetheless, ITU favours the latter to be implemented within $3-490 \mathrm{kHz}$ frequency for low speed transmission as mentioned in Table 1.

In order to work efficiently, CENELEC has several internal groups performing specific tasks. For instance, EN50065-1 is focussed on general requirements, frequency bands and electromagnetic disturbances for signalling on low-voltage electrical lines. EN50065-1 has subgroups which deal mainly with narrowband PLC, whereas EN55022 standard works on high-speed PLC in the spectrum of frequency between 150 to $500 \mathrm{kHz}$. While IEEE 1901.1 standard focuses on access communication systems as patented by IEEE. IEEE 1901.1 is designed for control transmissions of frequencies less than $15 \mathrm{MHz}$ in smart grid applications $[3,16]$ and [references therein].

These standards also specify signal coding and modulation techniques. IEEE 1901.2 is a revised version of the first one that advocates for use of Reed Solomon (RS) and convolutional codes (CC). The latter is also compatible with Internet Protocol version 6 (IPv6) [18]. ITU and

Table 1 Categories of PLC technology

\begin{tabular}{llc}
\hline Type of PLC & Features & Related works \\
\hline Ultra-narrowband & Frequency: $0.3-3 \mathrm{kHz}$ & {$[8,15]$} \\
& Designed for single way communication links & \\
& Data rate: Approx. $100 \mathrm{bps}$ & {$[15-17]$} \\
Narrowband & Frequency: $3-500 \mathrm{kHz}$ (Europe) & \\
& $10-490 \mathrm{kHz}$ (USA) & \\
& Designed for in-door communication links & \\
& Data rate: Approx. up to 100 kbps & {$[3,6,15-17]$} \\
Broadband & Frequency: $1.8-250 \mathrm{MHz}$ & \\
& Designed for in-home links & \\
& Data rate: Approx. up to 200 Mbps & \\
\hline
\end{tabular}


Table 2 Regulator standards for narrowband PLC and relevant modulation methods

\begin{tabular}{lll}
\hline Standards & Modulation method & Related works \\
\hline IEEE P1901.1 & OFDM & {$[19,20]$} \\
IEEE P1901 & OFDM & {$[19,21]$} \\
IEEE P1901.2 & PSK \& QAM (subclasses) & {$[19,22,23]$} \\
ITU-T G.9904 & OFDM & {$[19,24,25]$} \\
ITU-T G.9903 & OFDM & {$[19,25,26]$} \\
IEC61334 & S-FSK & {$[19,27-30]$} \\
G3-PLC & OFDM & {$[19,31,32]$} \\
ISO/IEC 14,908-1 & BPSK & {$[19,28,33,34]$} \\
PRIME & OFDM & {$[19,31,35]$} \\
ANSI/IEA 790.1,2 & BPSK & {$[19,36]$} \\
MAX2990 & OFDM & {$[19,37,38]$} \\
STM7580 & BPSK & {$[19]$} \\
\hline
\end{tabular}

Table 3 Regulator standards for broadband PLC and relevant modulation methods

\begin{tabular}{lll}
\hline Standards & Modulation method & Related works \\
\hline KNX & S-FSK & {$[18,19]$} \\
HomePlug 1.0 & D4PSK & {$[19,39]$} \\
HomePlug AV & BPSK, 4PSK, QAM \& subclasses & {$[19,40]$} \\
\hline
\end{tabular}

IEC have also contributed widely in developing some of these standards for narrowband PLC. Tables 2 and 3 provide summary lists of regulatory standards with regard to electrical power line communication technology and respective modulation techniques.

\section{Challenges facing PLC network}

Electrical power supply networks do offer an economical opportunity to realise infocommunication network at no extra cost of laying new communication cable lines. They also cover large areas thus increasing the size of communication network.

However, electrical lines are by design not meant for communication purposes. Such lines have unconducive features for transmission of signals. At the same time, power cables are the most asymmetrical network with many irregular connections extending between end-users and communication backbone lines [17, 41]. Continuous connection or disconnection of electrical appliances, such as, all switching changes, may change the topology [41].Each load switched and connected to the network operate at varying frequencies thereby injecting noises and imbalance impendance. Noise is discussed in the next section.

Güzelgöz et al. in [42] have comprehensively compared wireless and PLC transmission channel. From their comparison, it is evident that PLC is characterised by multipath propagation. These paths are caused by the presence of reflection points with different characteristic impedances. In [43], other factors that affect signal transmission such as frequency- selectivity of PLC channel, mismatched connections and branches have been reported. 
Attenuation which increases with frequency is another factor that hinders full exploitation of PL for communication use. Attenuation in this case depends on the length of branches, the material of the line and varying characteristic impedance too [44], and [references herein].

Electromagnetic compatibility is another challenge. From Maxwell's equation and electromagnetic theories $[45,46]$, and [references herein] electrical power lines, by extension PLC, act as antenna that excite electromagnetic waves. Therefore, use of frequency spectrum ranging to $30 \mathrm{MHz}$ which is reserved for radio communication may be interfered with by power line communication networks. As discussed under standards, there regulations have been set to minimise PLC electromagnetic interference on other services. Additionally, electromagnetic compatibility restrictions limit transmission power which in turn leads to low SNR at the receiver. Works on the latter are also ongoing.

The other major factor that degrades signal transmission in PL channels is non-Gaussian noise. This noise will be discussed in a section below. Before that, we describe PLC channel briefly.

\section{PL transmission channel}

In both [42] and [43], and [references herein] have compared power line communication channel as a multipath transmission medium. This is because of numerous reflection points, discrete and mismatched electrical loads in the PLC network. The channel load is either varying periodically or aperiodically due to connection or disconnection of electrical loads at varying times.

Understanding the channels behaviour is very vital in designing communication systems. Therefore, engineers and researchers apply channel models in order to characterise PLC transmission channel. Generally, there are two models: bottom-up and up-bottom approaches [43] and [references herein]. These approaches are iterative. Their approach includes both measurements and CAD simulations. Both approaches are either applied in time or frequency domain. The bottom-up model approach involves the use of mathematical model to define electrical power line channel. Model's parameters are calculated followed by simulation. To validate simulation results, measurements must be carried. The difference is that, the second approach starts with measurements. Thus, there is need for comprehensive information of the network and its physical topology. It also requires knowledge on impedances and features of cables. Up-bottom approach is practical and realistic. It is therefore the frequently used model by communication engineers. Mathematical models for both approaches are available in [19] and [references herein] for the reader to explore.

Berger et al. in [5] introduce the concept of MIMO PLC model. This is another technique that can be used to study the channel's feature in order to improve PL communication system's performance. Intensive studies of various models are focussed on possible exploitation of adaptive filters and scheduling, development of efficient PLC code, widening the bandwidth amongst others. Other models of study are mentioned in [47]. 


\section{Noise in PL communication system}

This noise can be defined based on its magnitude, origin and its representation main in time domain. Electrical power networks are heavy loaded in the so-called "last mile" and in-door areas. At these points, electrical appliances are the main source of noise in PL systems.

Noise in PL communication systems is non-Gaussian as opposed to traditional data communication channels which can be expressed in terms of additive white Gaussian noise $[48,49]$, and [references therein]. Several researches have grouped noise in PLC as background noise and impulsive noises. Background noise is subdivided into coloured background and narrowband noises, while impulsive noise, namely periodic asynchronous impulsive noise to the main lines frequency, periodic impulsive noise synchronous to the main lines frequency and aperiodic impulsive noise. The sources and forms of these noises have been widely studied and documented in [19] and [references therein]. Figures 2 and 3 show PLC system and noise in the channel.

\section{Modulation techniques for PLC systems}

Impulsive noise has great negative effect on transmitted signal. Therefore, in order to combat its effect multicarrier modulation techniques such as OFDM have superiority over single-carrier types.

Hrasnica et al. in [17] and Guzelgoz et al. in [42] have detailed explanations how other modulation used in wireless communication can be applied in PL communication system. Such modulation techniques include frequency hopping of spread spectrum family and random packet modulations. All these modulation techniques advocate for interleaving that spreads bit and symbol errors [50], and [references herein]. There is need though to keep guard with regard to intercarrier interferences.

Coding techniques such as turbo codes [51] and Solomon Reeds amongst others are also used to improve channel capacity. In [19], a brief and clear explanation on modulation and coding techniques has been presented. In Tables 2 and 3, recommended modulation techniques used in PLC are presented. In Table 4, we tabulate history of OFDM which has shown resistance to the effects of impulsive noise.

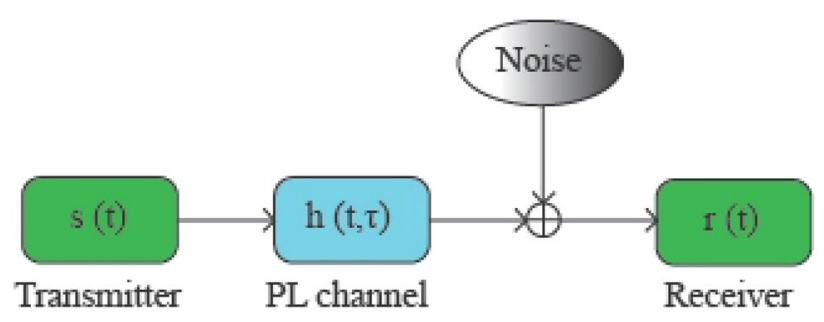

Fig. 2 PL Communication system 


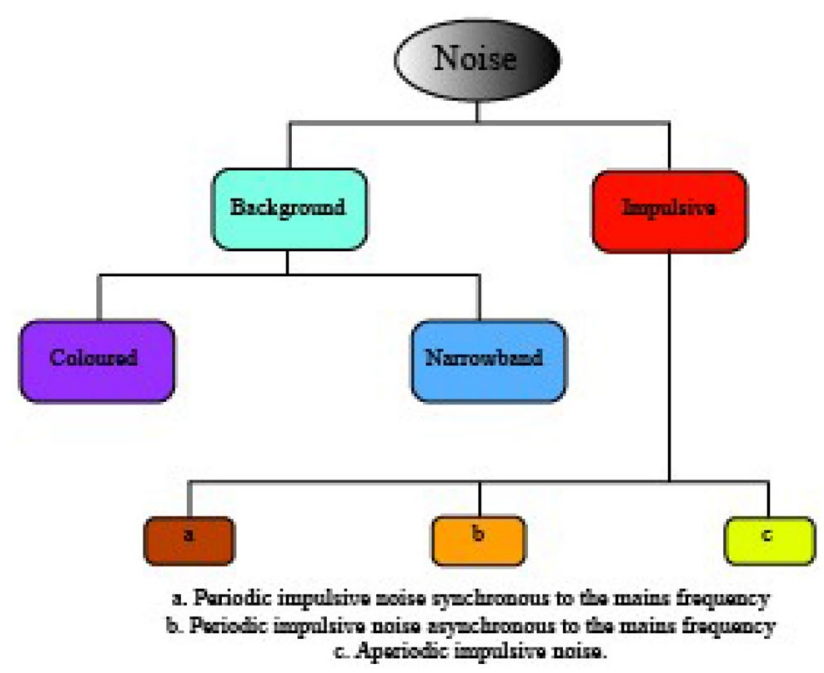

Fig. 3 PL Communication noise

Table 4 History of OFDM

\begin{tabular}{lll}
\hline Year & Description & Related works \\
\hline 1966 & Proposal of the first OFDM. This was for fading communication channels & {$[52-54]$} \\
1967 & Multicarrier systems & {$[53-55]$} \\
1970 & OFDM is patented & {$[53,54,56]$} \\
1971 & First application of DFT in OFDM & {$[53,54,57]$} \\
1980 & OFDM is applied in telephone networks & {$[53,54,58]$} \\
1985 & OFDM studies in mobile communication & {$[53,54,59]$} \\
1987 & Used in digital broadcasting & {$[53,54,60]$} \\
1991 \& 1994 & Report and standard for ADSL and HDSL & {$[53,54,61-63]$} \\
1995 & First standard for OFDM-based digital broadcasting system & {$[53,54,64]$} \\
1996 & WLAN standard & {$[53,54,65]$} \\
1997 & DVB-T standard & {$[53,54]$} \\
1998 & VDSL and BRAN standard & {$[53,54]$} \\
$1999 \&$ 2000 & IEEE 802.11a \& 802.11g WLAN standard & {$[53,54]$} \\
2003 & Wavelet-OFDM for high-speed PLC & {$[53,54]$} \\
2004 & IEEE 802.16 WMAN, 802.11n next generation WLAN and 802.15.3a MB-OFDM & {$[53,54]$} \\
2010 & Performance comparison between wavelet-OFDM \& filtered-OFDM & {$[53,54]$}
\end{tabular}

\section{Conclusion}

As human population continues to increase, the thirst and demand for data information and education will always be on the rise. The emergence of COVID19 has also put pressure on the existing communication systems as many organisations shift to "work from home". This calls for alternative signal transmission technology. Electrical power lines for communication have proven to be this option. With roughly $80 \%$ of the world population connected to the electric grid, this technology ready infrastructure to connect millions to info-communication network for business, education and other purposes. Thus, geographical communication coverage is increased. Despite 
the tremendous work done in both research industries and universities, there is need for more to be done to improve this technology.

In order for PLC to reach optimal maturity and mass implementation, the challenging obstacles discussed above must be tackled scientifically, properly and effectively. Additionally, there is need for adequate research funding and implementation of national regulation standards to comply with the international ones for uniformity across the globe. The latter issues affect developing countries such as Kenya and Türkiye.

In this paper, we have provided a detailed overview on the current state of PL Communication. We have summarised classes of PLC, regulation standards, transmission channel models, noises and tabulated modulation techniques. Although this field of research is maturing slowly and is fairly recent, it has become a promising one for near-future applications especially in Access Mode "Last mile" implementation. We hope this work will motivate researchers further to advance the penetration of PLC technology in developing countries' remote areas where electricity access is available but with rugged terrains.

\section{Abbreviations}

PLC: Power line communication; ITU: International Telecommunication Union; CENELEC: European Committee for Electro-technical Standardisation; IEC: International Electro-technical Commission; IEEE: Institute of Electrical and Electronics Engineer; ISO: International Organisation for Standardisation; FCC: Federal Communication Commission; RS: Reed Solomon; CC: Convolutional codes; IPv6: Internet Protocol version 6; CAD: Computer-aided design; MIMO: Multiple-input and multiple-output; SNR: Signal-to-noise ratio; OFDM: Orthogonal frequency division multiplexing; BSPK: Binary phase shift keying; QAM: Quadrature amplitude modulation; FSK: Frequency shift keying; DFT: Discrete Fourier transform; KNX: Konnex protocol; WLAN: Wireless local area network; PL: Power line; S-FSK: Spread FSK.

\section{Acknowledgements}

Not applicable.

\section{Authors' contributions}

The details of contributions of the authors in the present works are as follows: (a) AN contributed to the literature collection and manuscript preparation. (b) $\mathrm{IHC}$ contributed and edited the manuscript. Finally, all authors read and approved the final manuscript.

\section{Funding}

No funding organization is involved in this work.

\section{Availability of data and materials}

The paper presents original review work not previously published in similar form and not currently under consideration by another Journal.

\section{Declarations}

\section{Competing interests}

The authors declare that they have no competing interests.

\section{Author details}

${ }^{1}$ Department of Electrical and Electronics Engineering, Karadeniz Technical University, Trabzon, Turkey. ${ }^{2}$ Department of Electrical and Electronics Engineering, Machakos University, Machakos, Kenya.

Received: 30 December 2019 Accepted: 14 February 2021

Published online: 29 March 2021

\footnotetext{
References

1. Pal BP (1992) Fundamentals of fibre optics in telecommunication and sensor systems. Bohem Press, Zürich

2. Agrawal GP (2012) Fiber-optic communication systems, vol 222. Wiley, Hoboken

3. Yousuf MS, El-Shafei M (2007) Power line communications: an overview-part I. In: 2007 innovations in information technologies (IIT), pp 218-222

4. Carcelle X (2009) Power line communications in practice. Artech House, Norwood
} 
5. Berger LT, Schwager A, Pagani P, Schneider D (2014) MIMO power line communications: narrow and broadband standards, EMC, and advanced processing. CRC Press, Boca Raton

6. Gridley JH (2014) Principles of electrical transmission lines in power and communication: the commonwealth and international library: applied electricity and electronics division. Elsevier, Amsterdam

7. Mlynek P, Koutny M, Misure J (2008) The communication unit for remote data acquisition via the Internet. In: WSEAS international conference. proceedings. mathematics and computers in science and engineering

8. Schwartz M (2009) Carrier-wave telephony over power lines: early history [history of communications]. IEEE Commun Mag 47(1):14-18

9. Galli S, Scaglione A, Wang Z (2011) For the grid and through the grid: the role of power line communications in the smart grid. Proc IEEE 99(6):998-1027

10. Shaver DP (2011) Narrowband PLC solutions for AMl achieve long distance communications and flexibility with immediate market impact. In: IEEE international conference on consumer electronics (ICCE), pp 601-602

11. Antoniali M, De Piante M, Tonello AM (2013) PLC noise and channel characterization in a compact electrical car. In: 2013 IEEE 17th international symposium on power line communications and its applications, pp 29-34

12. Aalamifar F, Hassanein HS, Takahara G (2012) Viability of powerline communication for the smart grid. In: 2012 26th Biennial symposium on communications (QBSC), pp 19-23

13. Sittoni A, Brunelli D, Macii D, Tosato P, Petri D (2015) Street lighting in smart cities: a simulation tool for the design of systems based on narrowband PLC. In: 2015 IEEE first international smart cities conference (ISC2), pp 1-6

14. Mlynek P, Misurec J, Kolka Z, Slacik J, Fujdiak R (2015) Narrowband power line communication for smart metering and street lighting control. IFAC-Paper 48(4):215-219. https://doi.org/10.1016/j.ifacol.2015.07.035

15. Yigit M, Gungor VC, Tuna G, Rangoussi M, Fadel E (2014) Power line communication technologies for smart grid applications: a review of advances and challenges. Comput Netw 70:366-383

16. Shongwe T, Speidel U, Swart TG, Ferreira HC (2011) The effect of hamming distances on permutation codes for multiuser communication in the power line communications channel. In: IEEE Africon'11, pp 1-5

17. Hrasnica H, Haidine A, Lehnert R (2004) Broadband powerline communications networks. Wiley Online Library, Hoboken

18. Hallak G, Bumiller G, Lampe L, Tonello A, Swart T (2016) PLC for home and industry automation. In: Hallak G, Bumiller $\mathrm{G}$ (eds) Power line communications: principles, standards and applications from multimedia to smart grid. Wiley, Hoboken, pp 449-472

19. Ndjiongue A, Ferreira H (2019) Power line communications (PLC) technology: More than 20 years of intense research. Trans Emerg Telecommun Technol. https://doi.org/10.1002/ett.3575

20. Razazian K, Yazdani J (2011) CENELEC and powerline communication specification in realization of smart grid technology. In: 2011 2nd IEEE PES international conference and exhibition on innovative smart grid technologies, pp 1-7

21. Galli S, Logvinov O (2008) Recent developments in the standardization of power line communications within the IEEE. IEEE Commun Mag 46(7):64-71

22. Leclare J, Niktash A, Levi V (2017) An overview, history, and formation of IEEE P1901.2 for narrowband OFDM PLC. Maxim Integr Appl Note 5676:1-7

23. Logvinov O (2013) Netricity PLC and the IEEE P1901.2 Standard. HomePlug Power Line Alliance

24. Sanz A (2013) 3: ITU-T G. 9904 (PRIME 1.4)

25. Galli S, Lys T (2015) Next generation narrowband (under $500 \mathrm{kHz}$ ) power line communications (PLC) standards. China Commun 12(3):1-8

26. Van Laere A, Wawrzyniak C, Bette S, Moeyaert V (2016) Development, validation and utilization of an ITU-T G. 9903 PHY simulator for communication performance evaluation. In: 2016 international symposium on power line communications and its applications (ISPLC), pp 167-172

27. LuX, Monnier O, Dallas P (2012) Developing robust power line communications (PLC) with G3. Texas Instruments

28. I.-I. E. Commission and others (1996) IEC 61334-4-41

29. British Standards Institution (1998) Distribution Automation Using Distribution Line Carrier Systems: General considerations, guide for specification. BSI, London

30. Bali MC, Rebai C (2014) S-FSK modem design and experimental validation for robust narrowband powerline communication. In: 2014 IEEE international instrumentation and measurement technology conference (I2MTC) proceedings, pp 1251-1255

31. Hoch M (2011) Comparison of PLC G3 and PRIME. In: 2011 IEEE international symposium on power line communications and its applications, pp 165-169

32. Razazian K, Umari M, Kamalizad A, Loginov V, Navid M (2010) G3-PLC specification for powerline communication: overview, system simulation and field trial results. In: ISPLC2010, pp 313-318

33. San-Salvador Á, Herrero Á (2012) Contacting the devices: a review of communication protocols. In: Ambient intelligence-software and applications. Springer, pp 3-10

34. D'Innocenzo F, Bucci G, Fiorucci E, Ciancetta F (2017) Domestic electrical standard system for power line communication tests. In: 2017 IEEE international instrumentation and measurement technology conference (I2MTC), pp 1-6

35. Lu X, Kim IH, Vedantham R (2013) Implementing prime for robust and reliable power line communication (PLC). Texas Instruments White Paper

36. Podhoranský P, Lipovský M, Zemanovič J, Sabo M (2007) Transfer and error rate measurement in the PLC LonWorks systems

37. Yuan Z, Feng X, Li Y, Zhen Y (2010) Design of OFDM power line carrier communication node based on MAX2990. J Chengde Pet Coll (3), 14

38. Guo J, Su S, Fu C (2011) Research on power quality monitoring system based on low-voltage PLC technology. In: 2011 international conference on advanced power system automation and protection, vol 2, pp 1574-1577

39. Lee M, Newman RE, Latchman HA, Katar S, Yonge L (2003) HomePlug 1.0 powerline communication LANs_-protocol description and performance results. Int J Commun Syst 16(5):447-473 
40. Afkhamie KH, Katar S, Yonge L, Newman R (2005) An overview of the upcoming HomePlug AV standard. In: International symposium on power line communications and its applications, 2005, pp 400-404

41. Zimmermann M, Dostert K (2000) Low voltage power distribution network as last mile access network- signal propagation and noise scenario in the HF-range. AEU-ARCH Elektr Ubertragungstechn 54(1):13-22

42. Güzelgöz S, Arslan H, Islam A, Domijan A (2011) A review of wireless and PLC propagation channel characteristics for smart grid environments. J Electr Comput Eng 2011:1-12. https://doi.org/10.1155/2011/154040

43. Zimmermann M, Dostert K (2002) A multipath model for the powerline channel. IEEE Trans Commun 50(4):553-559

44. Durbak DW, Stewart JR (1990) PLC signal attenuation in branched networks. IEEE Trans Power Deliv 5(2):878-883

45. Harrington RF (1961) Time-harmonic electromagnetic fields. McGraw-Hill, New York

46. Balanis CA (2016) Antenna theory: analysis and design. Wiley, Hoboken

47. Ndolo AO (2016) Optimal narrowband and broadband data communication transmission in low-voltage power line. Int J Res Eng Technol 05(05):305-308. https://doi.org/10.15623/ijret.2016.0505056

48. Albert AL (1940) Electrical communication. Wiley, Hoboken

49. Albert AL (1952) The electrical fundamentals of communication. McGraw-Hill, New York

50. Al-Mawali KS, Hussain ZM (2009) Performance of bit-interleaved coded OFDM in power line communications with impulsive noise. In: 2009 international conference on advanced technologies for communications, pp 49-53

51. Abd-Alaziz W, Mei Z, Johnston M, Le Goff S (2017) Non-binary turbo-coded OFDM-PLC system in the presence of impulsive noise. In: 2017 25th European signal processing conference (EUSIPCO), pp 2576-2580

52. Chang RW (1966) Synthesis of band-limited orthogonal signals for multichannel data transmission. Bell Syst Tech $J$ 45(10):1775-1796

53. Hanzo L, Keller T (2007) OFDM and MC-CDMA: a primer. Wiley, Hoboken

54. Hanzo L, Webb W, Keller T (2000) Single-and multi-carrier quadrature amplitude modulation: principles and applications for personal communications, WATM and broadcasting: 2nd. IEEE Press-John Wiley, Hoboken

55. Saltzberg B (1967) Performance of an efficient parallel data transmission system. IEEE Trans Commun Technol 15(6):805-811

56. Chang RW (1970) Orthogonal frequency multiplex data transmission system. US Patent 3,488,445

57. Weinstein S, Ebert P (1971) Data transmission by frequency-division multiplexing using the discrete Fourier transform. IEEE Trans Commun Technol 19(5):628-634

58. Keasler WE, Bitzer DL, Tucker PT (1980) High speed modem suitable for operating with a switched network. Google Patents

59. Cimini L (1985) Analysis and simulation of a digital mobile channel using orthogonal frequency division multiplexing. IEEE Trans Commun 33(7):665-675

60. Lassalle R, Alard M (1987) Principles of modulation and channel coding for digital broadcasting for mobile receivers. EBU Tech Rev 224(1):68-190

61. Cioffi JM (1991) A multicarrier primer. In: ANSI T1E1, vol 4, pp 91-157

62. Veeneman D, Olshansky R (1995) ADSL for video and data services. In: Proceedings IEEE international conference on communications ICC'95, vol 2, pp 837-841

63. Taha HJ, Salleh M (2009) Multi-carrier transmission techniques for wireless communication systems: a survey. Wseas Trans Commun 8(5):457-472

64. EN ETSI (1995) ETS 300 401, Radio broadcasting systems: digital audio broadcasting (DAB) to mobile, portable and fixed receivers. European Telecommunications Standards Institute, Valbonne

65. Etsi E (1996) Radio equipment and systems (RES): high performance radio local area network (HIPERLAN) type 1; functional specification. ETSI ETS 300:652

\section{Submit your manuscript to a SpringerOpen ${ }^{\circ}$ journal and benefit from:}

- Convenient online submission

- Rigorous peer review

- Open access: articles freely available online

High visibility within the field

- Retaining the copyright to your article

Submit your next manuscript at $\boldsymbol{~ s p r i n g e r o p e n . c o m ~}$ 